\title{
Deceived bilateral filter for improving the automatic cell segmentation and tracking in the NF-kB pathway without nuclear staining
}

\author{
Aránzazu Sáenz ${ }^{1}$, Saúl Calderón ${ }^{1}$, Jorge Castro ${ }^{2}$, Rodrigo Mora ${ }^{3}$ and Francisco Siles ${ }^{1}$ \\ ${ }^{1}$ Universidad de Costa Rica/ PRIS-LAB: Pattern Recognition and Intelligent Systems Laboratory \\ Researcher, San José, Costa Rica \\ ${ }^{2}$ Universidad de Costa Rica/Electrical Engineering school, Electrical Engineering Master's student, San José, Costa Rica \\ ${ }^{3}$ Universidad de Costa Rica/Centro de Investigaciones en Enfermedades Tropicales CIET, Researcher, San José, Costa Rica
}

\begin{abstract}
In this work we implement an automatic videobased online cell tracking system for $\mathrm{TNF} \alpha$ treated cells in the transcription factor NF-kB pathway, with the aim to provide $p 65$ measurement approximation with time and individual cell resolution. The video used to test our proposed tracking system consists on cells transfected with fluorescent $p 65$ protein. Such protein switches its location within the cell, among the nucleus and cytoplasm over time, causing important changes on its apparent morphology. This represents a major challenge for an automatic cell tracking system. Additionally, images in the tested microscopy footage present noise and contrast variations. For noise reduction, we modified the deceived bilateral filter (DBF), a denoising and edge sharpening non linear filter. The proposed modification of the DBF consists in an adaptive border enhancement. We tested the cell tracking algorithm with different preprocessing configurations achieving a maximum tracking accuracy improvement of $28 \%$, with the use of the modified DBF.
\end{abstract}

Keywords - Online tracking, DBF, automatic cell activity analysis, NF-kB pathway, transcription factors.

\section{INTRODUCTION}

Transcription factors (TF) tightly regulate gene expression and are involved in many cellular processes, like cell cycle, adaptability, and metabolism. In the case of transcription factor NF-kB, it is known to be altered in numerous diseases, including cancer [1]. Hence, this pathway has been an interesting target for investigating such diseases.

NF-kB is regulated through a proteic inhibitor named Inhibitor of Kappa B (IkB), which binds to NF-kB and covers its nuclear localization signal, thus keeping NF-kB in the cytoplasm. When the NF-kB pathway is activated, IkB is marked for degradation, thus freeing NF-kB nuclear localization signal and allowing it to get translocated to the nucleus, where it can act as a TF for several genes involved in proliferation, survival, metastasis, angiogenesis and inflammation [1]. Since its activity depends upon which cellular compartment it is occupying, and it fluctuates over time, video anal- ysis with single-cell, compartment (nucleus and cytoplasm) and time resolution is necessary. The resulting quantitative data, as $p 65$ concentration (an active subunit of NF-kB), enables proper study of this and many other TF's alterations, with more precision in contrast of typical qualitative data analysis.

Time lapse fluorescence microscopy is a non-invasive method for studying TF's location, since it allows molecular biologists to locate proteins within cell's compartments in time, with individual cell resolution. In the case of this study, cells transfected with NF-kB-GFP were treated with TNF $\alpha$, and the cell's individual nucleus and cytoplasmatic concentrations of $p 65$ were observed over time.

Usually nuclei are used to track the cell as a primary structure, since it is less prone to shape changes over time and occlusion, unlike cell's cytoplasm. Hence, nuclei staining is usual. However, most of nuclear dyes used for staining can cause cellular stress [2], and could lead to biased results. This is the main reason a method in which nuclear staining is not required would be a very important research and clinical use tool. In the work presented in this paper the nuclei staining is not necessary.

The proposed cell tracking solution in this paper aims to provide a tool to automatically track cells on the NF-kB pathway and measure the presence of $p 65$ with cell, time and compartment resolution, requiring no user initialization. The approximation of $p 65$ presence within a cell compartment is defined as the average pixel value in the corresponding region in the image. In this paper we focus in the cell segmentation and tracking algorithm challenges, in order to enable future work focused on $p 65$ measurement.

The microscopy footage $V(t)$ used to test the system was taken from [3]. It presents poor contrast quality, fluctuant contrast and speckle noise, as seen in Figure 1.

The most challenging task is the variable cell's apparent morphology. Such behaviour is present since the fluorescence sampled by the microscopy's camera is given by the TF $p 65$ protein, which presents a density fluctuation at the cytoplasm and the nucleus compartments of every cell. 


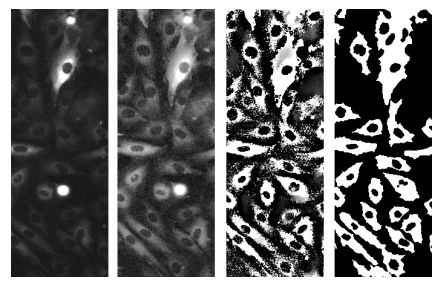

Fig. 1: From left to right: Original input image with cells transfected with $p 65-G F P$, CLAHE result, DBF result and final segmentation result (local Otsu thresholding and spurious objects removal)

We summarize the principal characteristics observed in the input data as follows:

- Images with noise and variable contrast along regions.

- A variable morphology of the cell's cytoplasm and nucleus, the objects of interest (OI), with important changes between frames.

- Slow and random movement of every OI.

- Low occlusion and OI's density.

Figure 1 shows a sample image with the aforementioned characteristics.

Based upon the previous itemized description, we detail the proposed method in Section III. Additionally, in this paper, we propose a modification for a denoising and border sharpening filter, the deceived bilateral filter (DBF), with the aim to improve image denoising and cell detection.

\section{PREVIOUS WORK}

The first and usual problem to deal in developing automatic cell activity tracking systems are noisy and poor contrast images. Basic approaches to diminish speckle noise are rank filters, low-pass filters and Gaussian or Butterworth based techniques. However the aforementioned approaches usually degrade the overall signal information. Hence more complex techniques for denoising are needed in order to avoid signal degradation.

The bilateral filter is a popular denoising technique which also preserves edges, performing Gaussian weighting in both spatial and range domains. In [4] an extensive review of the bilateral filter usage on microscopy imaging is done. In [5], the DBF, a novel bilateral filter based approach was proposed. The DBF improves edges acuity while performing moderate denoising, combining the bilateral filter with unsharped masking (USM) in a novel manner to avoid ringing. Even though, the DBF has been designed for image abstraction purposes in color images, its characteristics of moderate denoising and edge emphasis are useful to ease cell segmentation.
About the poor and variable contrast along an image issue, an usual approach is the use of adaptive histogram equalization techniques as the clip limited adaptive histogram equalization (CLAHE) [6]. An usual shortcoming of histogram based techniques is the amplification of noise.

As for the main cell segmentation techniques, based in [7], the approaches are grouped as thresholding based algorithms (Otsu, Kittler, locally adaptive modifications), template matching, watershed based techniques and deformable models (active contours). The advantages and shortcomings of each approach are also mentioned in [7].

For the specific case of cell segmentation in the NF-kB pathway, authors in [8] proposed an interactive cell segmentation by means of a geodesic commute distance and constrained density weighted Nystrom method. Despite its interesting results, manual initialization is required for every frame in this technique, making it unusable for our purposes.

Related to the later cell tracking stage, a deep analysis of the main techniques is to be found in [7]. Such approaches are classified in maximum likelihood algorithms, estimation based approaches and online cell tracking algorithms. Tracking systems implementations can combine techniques of these three approaches.

\section{PROPOSED METHOD}

The main workflow steps of the presented tracking system can be summarized as: noise reduction, initial cell segmentation, online cell tracking and NF-kB measurement approximation. For this paper we focus on the implementation and test of the first 3 stages. Based on the problem characterization and the previous work studied in Sections I and II we justify the implemented algorithms and show its results in this section.

\section{A Pre-processing}

In order to approximate the protein density of $p 65$ in both cytoplasm and nucleus of each cell a precise segmentation is needed, as stated in Section I. Hence handling noise is an important issue, in order to obtain better results in the detection of the cytoplasm and nuclei regions.

The pre-processing stage enhances the contrast and removes the noise of the initial image $I_{0}=V\left(t_{0}\right)$. To deal with poor and fluctuating contrast along the image, we use a contrast limited histogram equalization (CLAHE) as defined in [6]. The resulting image is shown on Figure 1.

However, the resulting image is still noisy. To diminish speckle noise we propose the use of the DBF [5]. The DBF 
is based on the original bilateral filter, combining it with an USM border enhancement method, as seen in equation 1 .

$$
\hat{f}_{\mathrm{DBF}}(p)=\left(\sum_{m}^{W} h(p, m)\right)^{-1}\left(\sum_{m}^{W} h(p, m) \hat{f}_{\mathrm{UM}}(m)\right)(1)
$$

where $h(p, m)$ corresponds to the bilateral filter kernel:

$$
h(p, m)=\exp \left(-\frac{(m-p)^{2}}{2 \sigma_{s}^{2}}\right) \exp \left(-\frac{(I(m)-I(p))^{2}}{2 \sigma_{r}^{2}}\right) .
$$

The USM resulting image $\hat{f}_{\mathrm{UM}}(p)$ is calculated as shown in equation 2 :

$$
\hat{f}_{\mathrm{UM}}=I+\lambda z
$$

where the laplacian image $z$ results from the convolution of the laplacian mask $L$ and the input image $I: z=L * I$. The amount of edge sharpening is defined by $\lambda$. To improve edge sharpening in a noisy and variable contrast input, we propose the use of an adaptive USM, calculating the edge sharpening factor according the local variance of the image $\sigma_{l}$. Hence, equation 2 is redefined as

$$
\hat{f}_{\mathrm{AUSM}}(p)=I(p)+\lambda\left(\sigma_{l}^{2}(p)\right) \ell(p)
$$

The function $\lambda\left(\sigma_{l}(p)\right)$ aims to amplify mid-contrast regions over high-contrast regions, and leave low-contrast unaltered, in order to avoid noise amplification. Hence, we define $\lambda\left(\sigma_{L}(p)\right)$ as a superposition of two trapezoid functions. The resulting image of performing both CLAHE and the modified DBF presents reduced noise and steeper edges, improving cell detection. Based on the definition of the cross bilateral filter in [9], the DBF can be expressed as $C B F\left[\hat{f}_{\mathrm{UM}}, I\right]$. As mentioned in [5], the DBF presents an accumulative result, thus applying it several times enhances the borders even further. Figure 1 shows the DBF results.

\section{B Cell segmentation}

To detect cells, Otsu local thresholding is performed. Local thresholding attenuates the effects of illumination variations in the image. The result is shown in Figure 1.

After Otsu thresholding, small spurious regions remain, and therefore are size thresholded. Also simple morphological operators are performed in order to discard spurious regions. This is performed for cytoplasm $(I(p)=1$ regions), nuclei and no cell regions $(I(p)=0)$. Cells might be detected with nucleus (primary structures) and without distinguishable nucleus (secondary structures). The segmentation results of the detected primary structures are shown in Figure 1.

\section{Cell tracking}

As mentioned in the enlisted characteristics of the input data in Section I the OIs presents important changes between frames, the use of a maximum likelihood approach based on image features (histograms of regions or borders) such as mean-shift, might not work properly. We also discarded the use of a estimation based approach like the Kalman filter, since OIs present almost no occlusion and its movement can be considered as noisy, hence lineal models might be too inaccurate. However, assuming small position changes and low OI's density is reasonable, given the observed input data, as stated in Section I. This justifies the use of a maximum likelihood approach for tracking based on spatial proximity.

More specifically, we propose the use of an online cell tracking approach, which initializes the segmentation window based on the previous OI's position found (defined by the nuclei centroids). A cell mask $M_{i, t}$ is computed to store nucleus and cytoplasm regions of the cell $i$ at time $t$. As for the first frame, the cell segmentation result $\mathrm{IS}_{0}$ of the pipelines described in Sub-sections A and B is used to detect the $i=1,2, \ldots, N$ primary structures. For each primary structure a mask $M_{i, 0}$, with a pixel centroid $p_{i, 0}$, is stored.

The search of the cell regions $M_{i, t}$ in the successive denoised frames $\mathrm{ID}_{t}$, for $t=1, \ldots, T$ is done in a window $W_{i, t}$ centered at pixel $p_{i, t-1}$. Cell segmentation is performed as detailed in Section B, resulting in $\mathrm{WS}_{i, t}$. The dimensions of the search window $W_{i, t}$ are defined by the sum of the cytoplasm size in pixels $c_{s i z e}$ and the mean centroid shift magnitude $\mu_{i, t-1}$ on the past $t-1$ frames. The set of primary structures $\left\{\mathrm{PS}_{i, t, j=1}, \mathrm{PS}_{i, t, j=2}, \ldots, \mathrm{PS}_{i, t, J}\right\}$ and secondary structures $\left\{\mathrm{SS}_{i, t, k=1}, \mathrm{SS}_{i, t, k=2}, \ldots, \mathrm{SS}_{i, t, K}\right\}$ found within $\mathrm{WS}_{i, t}$ are the track candidates for the cell mask $M_{i, t}$. Each track candidate is scored using a spatial proximity measure with a Gaussian kernel, resulting on the primary and secondary structures scores $S_{\mathrm{PS}}$ and $S_{\mathrm{SS}}$, respectively.

The maximum score $\max \left(S_{\mathrm{PS}}, S_{\mathrm{SS}}\right)$ defines the best tracking candidate, with $M_{i, t}=\mathrm{PS}_{i, t, j}$ when $\max \left(S_{\mathrm{PS}}\right) \geq \max \left(S_{\mathrm{SS}}\right)$ and $M_{i, t}=M_{i, t-1}$ otherwise. In both cases the centroid $p_{i, t-1}$ is updated to the new found centroid $p_{j}$ or $p_{k}$ respectively. Thus, when no primary structure is found within the search window, the centroid of the secondary structure found is used and the last primary structure mask found is updated. 


\section{EXPERIMENT RESULTS}

The video sequence used to test the proposed cell tracking algorithm corresponds to a record of the activity of 53 cells (excluding mitotic cells) in total, along 5 hours and 1 minute. The image resolution is $685 \times 445$ pixels, with 70 frames composing the sequence. For this paper, we used the tracking performance metrics suggested in [10]. The multiple object tracking accuracy (MOTA) and the multiple object tracking precision (MOTP) as proposed in [10], describes the overall accuracy and precision of the tracking algorithm, respectively. The test consists in running the tracking algorithm with different preprocessing algorithm configurations, in order to asses the impact of the DBF based filter for denoising and edge sharpening. The parameters of the denoising algorithms are identical along all the preprocessing algorithm configurations. The DBF was tested after 1, 2 and 3 iterations. The ground-truth for the test video sequence was generated with Sensarea v 1.2 [11].

Table 1: Cell tracking results, using different preprocessing configurations. (*)CLAHE used first. All the results are expressed in percent (\%)

\begin{tabular}{llllll}
\hline PP Confs. & FN & FP & MME & MOTP & MOTA \\
\hline No PP & 62.35 & 4.95 & 0.08 & 20.96 & 32.62 \\
BF* $^{*}$ & 53.20 & 3.20 & 0.02 & 19.6 & 43.57 \\
DBF* it. 1 & 33.86 & 9.79 & 0.18 & 21.64 & 56.16 \\
DBF* it. 2 & 30.66 & 8.44 & 0.15 & 21.91 & 60.74 \\
DBF* it. 3 & 32.64 & 14.13 & 0.29 & 21.31 & 52.93 \\
\hline
\end{tabular}

The results detailed in Table 1 reveals that the use of the DBF with two iterations has the most important impact in MOTA performance. Such improvement boosts cell detection, decreasing false negatives. Performing more iterations of the DBF (three iterations) causes previous spurious regions to be denoised by the DBF increasing false positives, resulting in an overall worse result. To address this, low contrast regions could be amplified less to avoid such phenomena in later iterations of the DBF.

\section{CONCLUSION AND FUTURE WORK}

A novel preprocessing algorithm for noisy and poor contrast cell images along with an online cell tracking pipeline in the NF-kB way, were proposed and tested in this paper. The results show a remarkable MOTA improvement with the use of the modified DBF. The proposed solution in this paper is useful for medicine and live science researchers as it provides automatic cell detection and tracking, allowing the generation of $p 65$ measurements, with no need of nuclear staining.
Empirical tests show that the accuracy of cytoplasm region detection is not acceptable, hence more research is needed to overcome such limitation. The quantified tracking results show that the algorithm provides acceptable nuclei detection, allowing the measurement of $p 65$ concentration within each nucleus. False negatives were lowered with the use of the DBF, as false positives did not increase significantly.

As future work more modifications to the DBF can be done in order to improve noise filtering performance, as the use of rank order filter modifications [5]. The proposed tracking algorithm is able to provide $p 65$ measurement with individual cell and time resolution, however $p 65$ measurement results must be compared against ground-truth data.

\section{CONFLICT OF INTEREST}

The authors declare that they have no conflict of interest.

\section{ACKNOWLEDGEMENTS}

The research presented in this paper is partially funded by the National Center of High Technology (CENAT), Costa Rica. We thank Andrés Mora for his support to this research.

\section{REFERENCES}

1. Ling J, Kumar R. Crosstalk between NFkB and glucocorticoid signaling: A potential target of breast cancer therapy Cancer Letters. 2012;322:119 - 126 .

2. Oh K, Bustin M, Mazur S J., Appella E, Kraemer K H.. UV-induced histone $\mathrm{H} 2 \mathrm{AX}$ phosphorylation and DNA damage related proteins accumulate and persist in nucleotide excision repair-deficient XP-B cells DNA Repair. 2011;10:5 - 15.

3. Tay S, Hughey J, Lee T, Lipniacki T, Quake S, Covert M. Single-cell NF-KB dynamics reveal digital activation and analogue information processing Nature. 2010;466:267-271.

4. Jiang W, Baker M, Wu Q, Bajaj C, Chiu W. Applications of a bilateral denoising filter in biological electron microscopy Journal of Structural Biology. 2003;144:114-122.

5. Calderon S, Siles F. Deceived Bilateral Filter for Improving the Classification of Football Players from TV Broadcast in IEEE 3rd International Conference and Workshop on Bioinspired Intelligence 2014.

6. Zuiderveld K. Graphics Gems IV in Graphics Gems IV (Heckbert Paul S.. , ed.)ch. Contrast Limited Adaptive Histogram Equalization, :474-485San Diego, CA, USA: Academic Press Professional, Inc. 1994.

7. Meijering E, Dzyubachyk O, Smal I. Methods for Cell and Particle Tracking Imaging and Spectroscopic Analysis of Living Cells. 2012;504:183-200.

8. Cheng-Jin D, Marcello M, Spiller D, White M, Bretschneider T. Interactive segmentation of clustered cells via geodesic commute distance and constrained density weighted Nyström method Cytometry Part A. 2010;77:1137-1147.

9. Kornprobst P, Tumblin J. Bilateral filtering: Theory and applications. Now Publishers Inc 2009. 
10. Bernardin K, Stiefelhagen R. Evaluating multiple object tracking performance: the CLEAR MOT metrics EURASIP Journal on Image and Video Processing. 2008;2008.

11. Bertolino P. Sensarea: An authoring tool to create accurate clickable videos in Content-Based Multimedia Indexing (CBMI), 2012 10th International Workshop on:1-4IEEE 2012. 